\title{
TUTORIAL ON QUASI-SPARSE EIGENVECTOR DIAGONALIZATION
}

\author{
DEAN LEE \\ University of Massachusetts, Amherst, MA 01003 USA \\ dlee@physics.umass.edu
}

We review several topics related to the diagonalization of quantum field Hamilto-
nians using the quasi-sparse eigenvector (QSE) method.

\section{Introduction}

Quasi-sparse eigenvector (QSE) diagonalization is a new computational method which finds the low-lying eigenvalues and eigenvectors for a general quantum field Hamiltonian ${ }^{1}$. It is able to handle the exponential increase in the size of Fock space for large systems by exploiting the sparsity of the Hamiltonian. QSE diagonalization can even be applied directly to infinitedimensional systems. The method is most effective when the splitting between low-lying eigenvalues is not too small compared to the size of the offdiagonal Hamiltonian matrix entries. In such cases the low-lying eigenvectors are quasi-sparse, meaning that the vector is dominated by a small fraction of its largest components. The QSE algorithm can then be applied to the Hamiltonian $H$ using the following steps:

1. Select a subset of orthonormal basis vectors $\left\{e_{i_{1}}, \cdots, e_{i_{n}}\right\}$ and call the corresponding subspace $S$.

2. Diagonalize $H$ restricted to $S$ and find one eigenvector $v$.

3 . Sort the basis components $\left\langle e_{i_{j}} \mid v\right\rangle$ according to their magnitude and remove the least important basis vectors.

4. Replace the discarded basis vectors by new basis vectors. These are selected at random from a pool of candidate basis vectors which are connected to the old basis vectors through non-vanishing matrix elements of $H$.

5. Redefine $S$ as the subspace spanned by the updated set of basis vectors and repeat steps 2 through 5 .

If the subset of basis vectors is sufficiently large, the exact eigenvectors will be stable fixed points of the update process.

guangzhou2000: submitted to World Scientific on November 10, 2018 
The purpose of this short tutorial is to provide additional information for those interested in writing or using codes that implement QSE methods. We discuss the calculation of Hamiltonian matrix elements in the momentum Fock space representation and the generation and selection of new basis states. We also include a simple program which applies the QSE method to find the ground state of an input matrix. Readers interested in an introductory overview of QSE diagonalization are encouraged to consult the references in 1.

\section{Fock states and the Hamiltonian matrix}

It is most effective to describe the details of a QSE calculation in the context of a specific example. In this discussion we consider $\phi^{4}$ theory in $1+1$ dimensions, the same example as in ${ }^{1}$. Some of the techniques described here have been specifically optimized for scalar field theories. Other systems require a somewhat different set of tools and will be discussed in the future.

The Hamiltonian density for $\phi^{4}$ theory in $1+1$ dimensions has the form

$$
\mathcal{H}=\frac{1}{2}\left(\frac{\partial \phi}{\partial t}\right)^{2}+\frac{1}{2}\left(\frac{\partial \phi}{\partial x}\right)^{2}+\frac{\mu^{2}}{2} \phi^{2}+\frac{\lambda}{4 !}: \phi^{4}:
$$

where the normal ordering is with respect to the mass $\mu$. We consider the system in a periodic box of length $2 L$. We then expand in momentum modes and reinterpret the problem as an equivalent Schrödinger equation ${ }^{2}$. We will use a momentum cutoff scheme where the modes $q_{n}$ are restricted in momentum so that $|n| \leq N_{\max }$. The resulting Hamiltonian is

$$
\begin{aligned}
H & =-\frac{1}{2} \sum_{n} \frac{\partial}{\partial q_{-n}} \frac{\partial}{\partial q_{n}}+\frac{1}{2} \sum_{n}\left(\omega_{n}^{2}(\mu)-\frac{\lambda b(\mu)}{4 L}\right) q_{-n} q_{n} \\
& +\frac{\lambda}{4 ! 2 L} \sum_{n_{1}+n_{2}+n_{3}+n_{4}=0} q_{n_{1}} q_{n_{2}} q_{n_{3}} q_{n_{4}},
\end{aligned}
$$

where

$$
\omega_{n}(\mu)=\sqrt{\frac{n^{2} \pi^{2}}{L^{2}}+\mu^{2}}
$$

and $b(\mu)$ is the coefficient for the mass counterterm

$$
b(\mu)=\sum_{n} \frac{1}{2 \omega_{n}(\mu)} .
$$

It is convenient to split the Hamiltonian into free and interacting parts with respect to an arbitrary mass $\mu^{\prime}$ :

guangzhou2000: submitted to World Scientific on November 10, 2018 


$$
\begin{aligned}
& H_{\text {free }}=-\frac{1}{2} \sum_{n} \frac{\partial}{\partial q_{-n}} \frac{\partial}{\partial q_{n}}+\frac{1}{2} \sum_{n} \omega_{n}^{2}\left(\mu^{\prime}\right) q_{-n} q_{n} \\
& H=H_{\text {free }}+\frac{1}{2} \sum_{n}\left(\mu^{2}-\mu^{\prime 2}-\frac{\lambda b(\mu)}{4 L}\right) q_{-n} q_{n} \\
& \quad+\frac{\lambda}{4 ! 2 L} \sum_{n_{1}+n_{2}+n_{3}+n_{4}=0} q_{n_{1}} q_{n_{2}} q_{n_{3}} q_{n_{4}} .
\end{aligned}
$$

$\mu^{\prime}$ will be used to define the basis states of our Fock space. Since $H$ is independent $\mu^{\prime}$, it is useful to perform calculations for different $\mu^{\prime}$ to obtain an estimate of the error. It is also helpful to find the range of values for $\mu^{\prime}$ which maximizes the quasi-sparsity of the eigenvectors. This can be used to improve the accuracy of the QSE calculation.

Let us define ladder operators with respect to $\mu^{\prime}$,

$$
\begin{aligned}
& a_{n}\left(\mu^{\prime}\right)=\frac{1}{\sqrt{2 \omega_{n}\left(\mu^{\prime}\right)}}\left[q_{n} \omega_{n}\left(\mu^{\prime}\right)+\frac{\partial}{\partial q_{-n}}\right] \\
& a_{n}^{\dagger}\left(\mu^{\prime}\right)=\frac{1}{\sqrt{2 \omega_{n}\left(\mu^{\prime}\right)}}\left[q_{-n} \omega_{n}\left(\mu^{\prime}\right)-\frac{\partial}{\partial q_{n}}\right] .
\end{aligned}
$$

These satisfy the usual commutation relations,

$$
\begin{aligned}
& {\left[a_{n}, a_{m}^{\dagger}\right]=\delta_{n m}} \\
& {\left[a_{n}, a_{m}\right]=\left[a_{n}^{\dagger}, a_{m}^{\dagger}\right]=0 .}
\end{aligned}
$$

The Hamiltonian can now be rewritten as

$$
\begin{aligned}
H & =\sum_{n} \omega_{n}\left(\mu^{\prime}\right) a_{n}^{\dagger} a_{n}+\frac{1}{4}\left(\mu^{2}-\mu^{\prime 2}-\frac{\lambda b(\mu)}{4 L}\right) \sum_{n} \frac{\left(a_{-n}+a_{n}^{\dagger}\right)\left(a_{n}+a_{-n}^{\dagger}\right)}{\omega_{n}\left(\mu^{\prime}\right)} \\
& +\frac{\lambda}{192 L} \sum_{n_{1}+n_{2}+n_{3}+n_{4}=0}\left[\frac{\left(a_{n_{1}}+a_{-n_{1}}^{\dagger}\right)}{\sqrt{\omega_{n_{1}}\left(\mu^{\prime}\right)}} \frac{\left(a_{n_{2}}+a_{-n_{2}}^{\dagger}\right)}{\sqrt{\omega_{n_{2}}\left(\mu^{\prime}\right)}} \frac{\left(a_{n_{3}}+a_{-n_{3}}^{\dagger}\right)}{\sqrt{\omega_{n_{3}}\left(\mu^{\prime}\right)}} \frac{\left(a_{n_{4}}+a_{-n_{4}}^{\dagger}\right)}{\sqrt{\omega_{n_{4}}\left(\mu^{\prime}\right)}}\right] .
\end{aligned}
$$

In (10) we have omitted constants contributing only to the vacuum energy.

We can represent any momentum-space Fock state as a string of occupation numbers,

$$
\left|O_{-} N_{\max }, \cdots, o_{N_{\max }}\right\rangle
$$

where

$$
a_{n}^{\dagger} a_{n}\left|o_{-N_{\max }}, \cdots, o_{N_{\max }}\right\rangle=o_{n}\left|o_{-N_{\max }}, \cdots, o_{N_{\max }}\right\rangle .
$$


From the usual ladder operator relations, it is straightforward to calculate the matrix element of $H$ between two arbitrary Fock states. In ${ }^{1}$ auxiliary cutoffs were used to render the dimension of Fock space finite. This is in fact an unnecessary restriction and QSE diagonalization encounters no difficulties dealing with the full infinite-dimensional space.

\section{New basis vectors}

Aside from calculating matrix elements, the only other fundamental operation involving basis vectors is the generation of new basis vectors. As mentioned before, the new states should be connected to an old basis vector through nonvanishing matrix elements of $H$. Let us refer to the old basis vector as $|e\rangle$. For this example there are two types of terms in our interaction Hamiltonian, a quartic interaction

$$
\sum_{n_{1}, n_{2}, n_{3}}\left(a_{n_{1}}+a_{-n_{1}}^{\dagger}\right)\left(a_{n_{2}}+a_{-n_{2}}^{\dagger}\right)\left(a_{n_{3}}+a_{-n_{3}}^{\dagger}\right)\left(a_{-n_{1}-n_{2}-n_{3}}+a_{n_{1}+n_{2}+n_{3}}^{\dagger}\right),
$$

and a quadratic interaction

$$
\sum_{n}\left(a_{-n}+a_{n}^{\dagger}\right)\left(a_{n}+a_{-n}^{\dagger}\right) .
$$

To produce a new vector from $|e\rangle$ we simply choose one of the possible operator monomials

$$
\begin{aligned}
& a_{n_{1}} a_{n_{2}} a_{n_{3}} a_{-n_{1}-n_{2}-n_{3}}, a_{-n_{1}}^{\dagger} a_{n_{2}} a_{n_{3}} a_{-n_{1}-n_{2}-n_{3}}, \cdots, \\
& a_{-n} a_{n}, a_{n}^{\dagger} a_{-n}^{\dagger}, \cdots
\end{aligned}
$$

and let it act upon $|e\rangle$. Our experience is that the interactions involving the small momentum modes are generally more important than those for the large momentum modes, a signal that the ultraviolet divergences have been properly renormalized. For this reason it is best to arrange the selection probabilities such that the smaller values of $\left|n_{1}\right|,\left|n_{2}\right|,\left|n_{3}\right|$ and $|n|$ are chosen more often.

We note that the new basis vector selection will occasionally fail. Either the vector is zero due to an annihilation operator acting on an unoccupied state or the vector is already in our subset of basis vectors and therefore not new. In either case we simply select a new basis vector again. If the selection process fails repeatedly then a different old basis vector $\left|e^{\prime}\right\rangle$ is used.

guangzhou2000: submitted to World Scientific on November 10, 2018 


\section{Sample code}

We have considered two basic operations, the calculation of matrix elements and the generation of new basis states. The subroutines which perform these tasks will depend on the form of the quantum field Hamiltonian. The main structure of the QSE algorithm, however, is independent of the details of the Hamiltonian. We demonstrate the essential features of the algorithm with the following short MATLAB program. In this example we find the ground state of a finite matrix. To keep the example as simple as possible, we avoid calculating matrix elements and generating new basis states by assuming that the entire Hamiltonian matrix can be stored in memory.

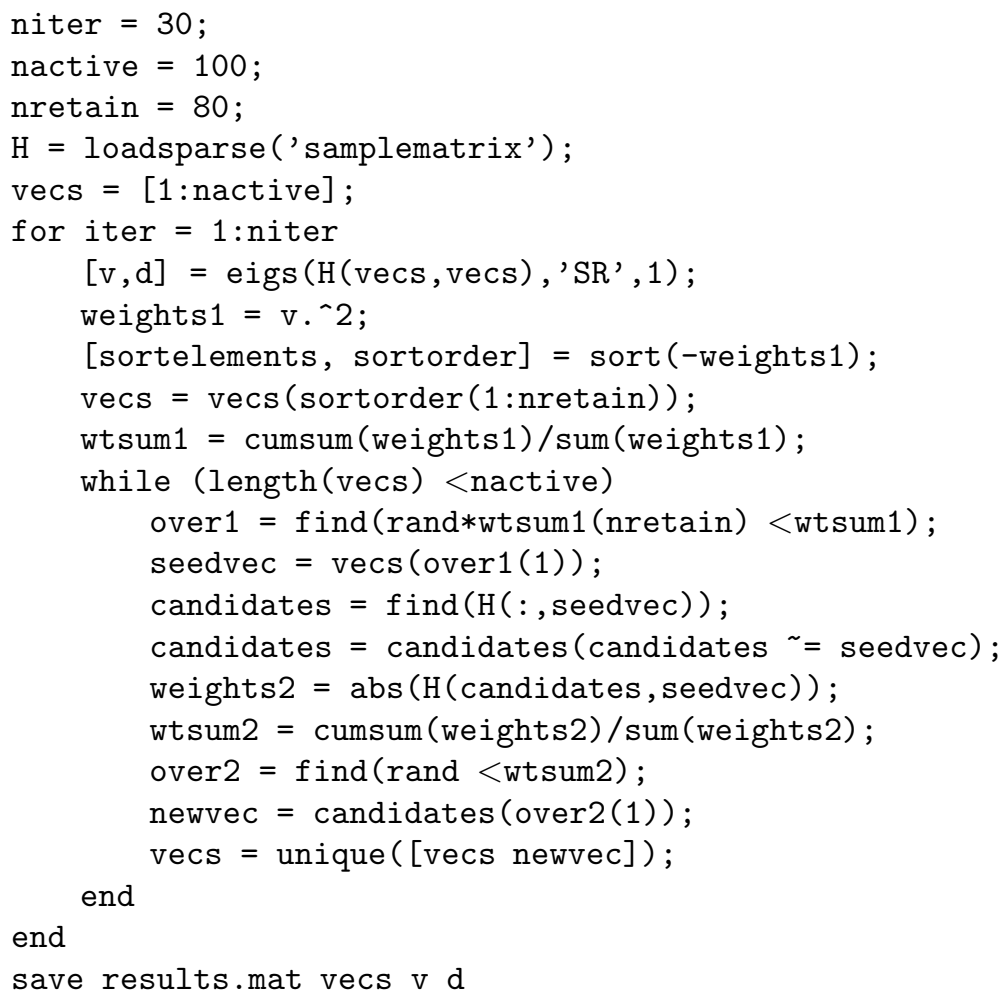

niter is the number of iterations of the algorithm. nactive is the number of basis states used to form the subspace $S$, and nretain is the number of basis states kept after removing the tail of the eigenvector distribution. loadsparse is a program which reads in a datafile called samplematrix. This 
data is stored in the matrix variable $\mathrm{H}$. The remaining code can be broken down in terms of the five basic steps of the QSE algorithm.

Select a subset of orthonormal basis vectors $\left\{e_{i_{1}}, \cdots, e_{i_{n}}\right\}$ and call the corresponding subspace $S$.

vecs $=[1$ :nactive $]$

The variable vecs is a row vector which labels the basis vectors in the subspace $S$. In our example vecs is initialized to be the first nactive basis vectors. We are assuming that the ground state of the system has a non-zero overlap with at least one of the first nactive basis vectors. A more general initialization would be to choose a random subset of basis vectors.

Diagonalize $H$ restricted to $S$ and find one eigenvector $v$.

$[\mathrm{v}, \mathrm{d}]=\operatorname{eigs}\left(\mathrm{H}(\mathrm{vecs}, \mathrm{vecs}),{ }^{\prime} \mathrm{SR}\right.$ ', 1$)$;

The function eigs is a sparse matrix Arnoldi diagonalization routine. It is being called with the parameters 'SR', 1 which tells eigs to find the eigenvalue and eigenvector with the smallest real part. The Hamiltonian submatrix corresponding with basis vectors in vecs is diagonalized. The eigenvector is stored in the column vector $\mathrm{v}$ and the eigenvalue is stored as the variable $\mathrm{d}$.

Sort the basis components $\left\langle e_{i_{j}} \mid v\right\rangle$ according to their magnitude and remove the least important basis vectors.

weights1 = v.`2;

[sortelements, sortorder] = sort (-weights 1$)$;

vecs $=\operatorname{vecs}($ sortorder $(1:$ nretain $))$;

weights 1 is a column vector which contains the squares of the components of the eigenvector v. sortelements is a list of the elements of -weights1 from smallest to greatest. sortorder is the corresponding list of locations for these elements. If for example weights $1=\left[\begin{array}{lll}.2 & .5 & .3\end{array}\right]$, then sortorder $=\left[\begin{array}{lll}2 & 3 & 1\end{array}\right]$. The list sortelements is not used. Only the nretain most important basis vectors are kept in vecs.

Replace the discarded basis vectors by new basis vectors. These are selected at random from a pool of candidate basis vectors which are connected to the old basis vectors through non-vanishing matrix elements of $H$.

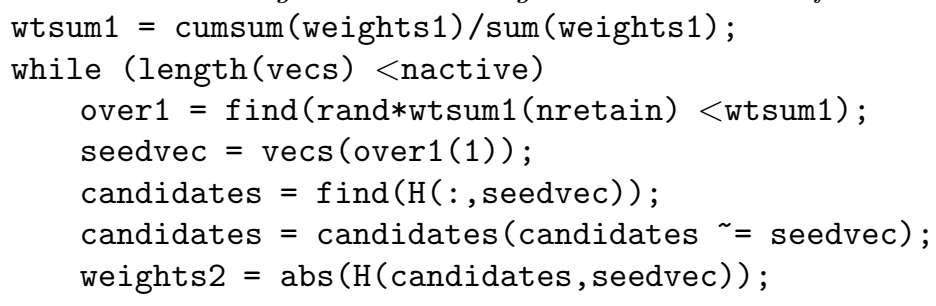

guangzhou2000: submitted to World Scientific on November 10, 2018 


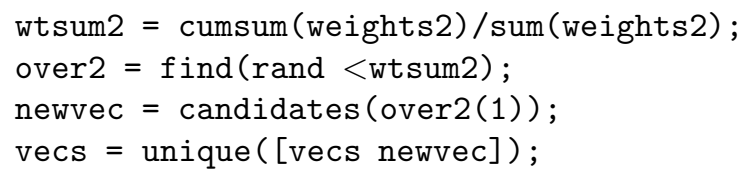

cumsum is a function which takes the input list and creates another list consisting of partial sums. wtsum1 is therefore an ascending string of numbers between 0 and 1 such that the difference between an entry and its preceding entry is proportional to the corresponding value of weights 1 . over 1 is a location list of elements of wtsum1 which are greater than the product of a generated random number between 0 and 1 and wtsum1 (nretain). over1(1) is the first entry of over1. We note that over1(1) must be less than or equal to nretain. seedvec is the basis vector which corresponds with over1(1). candidates is a column vector consisting of all basis vectors which have a non-zero matrix element with seedvec. Since the task is to find new basis vectors, seedvec is removed from candidates.

weights 2 is a column vector containing the absolute values of the matrix transition elements from seedvec. wtsum2 is an ascending string between 0 and 1 such that the difference between an entry and its preceding entry is proportional to the value of weights2. over 2 is a location list of elements of wtsum2 greater than a generated random number. newvec is the basis vector which corresponds with over2(1). newvec is appended to the set vecs. The function unique sorts the input list and removes any repetitions. This loop is repeated until vecs has nactive elements.

Redefine $S$ as the subspace spanned by the updated set of basis vectors and repeat steps 2 through 5 .

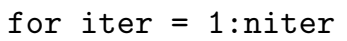

The algorithm is iterated niter times and the final results are saved in the file results.mat. More examples of QSE diagonalization codes for various field theory systems written in MATLAB, Fortran, and/or $\mathrm{C}++$ will be made available in the future.

\section{Summary}

In this short tutorial we have listed some supplemental material for those interested in writing or using codes that implement QSE methods. We have

guangzhou2000: submitted to World Scientific on November 10, 2018 
discussed the calculation of Hamiltonian matrix elements and the selection of new basis states. We have also presented a simple MATLAB program which applies the QSE method to find the ground state of a matrix.

The discussion here was limited to the basic QSE method. As presented in the Guangzhou workshop there have also been interesting new developments in QSE diagonalization regarding the technique of stochastic error correction. This technique will be presented in a forthcoming work ${ }^{4}$.

\section{Acknowledgments}

I thank my collaborators on the papers cited here and the organizers and participants of the International Workshop on Nonperturbative Methods and Lattice QCD in Guangzhou. Financial support was provided by the National Science Foundation.

\section{References}

1. D. J. Lee, N. Salwen, D. D. Lee, hep-th/0002251; D. Lee, hep-th/0003296.

2. P. Marrero, E. Roura, D. Lee, Phys. Lett. B471 (1999) 45.

3. N. Salwen, D. Lee, Phys. Rev. D62 (2000) 025006.

4. D. Lee, N. Salwen, M. Windoloski, work in progress. 